\title{
Fragmentación, espacio y rentismo en la neoliberalización de la salud: el caso bogotano*
}

\author{
Fragmentation, Space and Rentism in the \\ Neoliberalization of Health: the case of Bogotá
}

\author{
Edgar Valero-Julio ${ }^{1}$ \\ Universidad Nacional de Colombia, Bogotá, Colombia \\ eavaleroj@unal.edu.co \\ https://orcid.org/0000-0002-9538-1955 \\ Alice Beuf ${ }^{2}$ \\ Universidad Nacional de Colombia, Bogotá, Colombia \\ aabeuf@unal.edu.co \\ https://orcid.org/0000-0002-2181-1481 \\ Hans Rojas-Valencia ${ }^{3}$ \\ Universidad Nacional de Colombia, Bogotá, Colombia \\ horojasv@unal.edu.co \\ https://orcid.org/0000-0001-8907-5997 \\ Michael Tache-Victorino 4 \\ Universidad Nacional de Colombia, Bogotá, Colombia \\ mtachev@unal.edu.co \\ https://orcid.org/0000-0003-2640-0566
}

Recibido: 23-08-19

Aprobado: 10-06-20

\footnotetext{
* Este artículo es un producto final del proyecto de investigación “Turismo de salud en Bogotá: tendencias e impactos de un proceso global" que ha sido financiada mediante la convocatoria Fals Borda de apoyo a la investigación, de la Facultad de Ciencias Humanas de la Universidad Nacional de Colombia (sede Bogotá).

1 Doctor en Historia.

2 Doctora en Geografía humana, económica y regional.

3 Especialista en Métodos estadísticos básicos.

4 Politólogo.
} 


\section{Resumen}

El objetivo del presente artículo es analizar las lógicas de inversión y las modalidades de reconfiguración del sector salud en Bogotá. Las reformas neoliberales de los años 90, profundizadas por reglamentaciones específicas en la década de los 2000 , han transformado un sistema que nunca reconoció la atención universal como derecho ciudadano. A partir de entrevistas a diversos actores del sistema de salud, revisión bibliográfica, de prensa y de informes corporativos, evidenciamos cómo hoy conviven muy heterogéneas estructuras, desde ruinosos hospitales públicos, entidades rentistas que se apropian de recursos oficiales, y avanzadas clínicas proyectándose a la conformación de clústeres y turismo de salud. Mostramos cómo la coexistencia de varios modelos de negocio conlleva una problemática diferenciación de ámbitos de manejo laboral y modalidades de producción de ciudad.

Palabras clave: economía de la salud; globalización; concentración económica; condiciones de trabajo; renovación urbana.

\section{Abstract}

This paper aims to analyze the logic behind investment and the modalities of the reconfiguration of the Health area in Bogotá. The Neoliberal reforms of the 90's, deepen through specific regulations in the 2000's, has transformed a system that never acknowledged the universal attention as a civilian right. From interviews to different actors of the health system, bibliographic review, press and corporative reports, we can point how heterogeneous structures coexist, from ruined public hospitals, rentier entities that take over official resources, to advanced clinics that are projected towards to the conformation of clusters and health tourism. We show how the coexistence of different business models lead to a problematic distinction of scope of labor management and modalities of production of the city.

Keywords: health economy; globalization; economic concentration; labor conditions; urban renewal. 


\section{Introducción}

La planificación hospitalaria, que se desarrolló en la posguerra, en el marco de estados de bienestar keynesianos, estimuló la generación de un cuerpo de conocimientos teórico metodológicos, cuando médicos de salud pública, economistas, sociólogos y geógrafos de la salud, entre otros, se dieron a la tarea de identificar las necesidades de la población, para determinar los requerimientos de cada territorio en materia de prestación de servicios sanitarios. Así, la geografía de la salud se consolidó como una disciplina científica orientada a asesorar la formulación de políticas de salud en su dimensión territorial, en particular en Canadá, Reino Unido y Francia (Thouez, 1987; Eyles, 1987; Vigneron, 1999). Esta perspectiva no aplica para Colombia, dado que no se reconoce el principio de equidad territorial, según el cual deben ser más dotados los territorios con mayores necesidades (porque son más poblados o porque sus poblaciones son más envejecidas, o presentan cualquier otra especificidad).

El sistema colombiano no está orientado a atender las necesidades sanitarias de una población en un territorio dado, sino a garantizar la capacidad de acceso de los usuarios a un aseguramiento en salud bajo reglas de mercado. En este marco, y desde una perspectiva interdisciplinaria, queremos indagar acerca del proceso de neoliberalización del sistema de salud en Colombia y en el área metropolitana de Bogotá en particular. En ausencia de criterios de justicia espacial, nos interesa evidenciar las lógicas empresariales e inversionistas que generan negocios altamente rentables cuando la prestación de salud de por sí no lo es, y de qué modo producen ciudad y ámbitos institucionales notoriamente diferenciados.

Las transformaciones del sector salud en las últimas décadas, por estar ligadas a la adopción del modelo neoliberal, así como al influjo de tendencias de inversión y lógicas de negocio internacionalizadas, hacen parte de fenómenos de globalización. Si en un principio este proceso se interpretó como de posible homogeneización cultural y surgimiento de una civilización global, más adelante se valoraron los impactos diferenciados sobre territorios, poblaciones e instituciones. A distintos niveles resulta estimulada la fragmentación, sea por el surgimiento de nacionalismos y fundamentalismos en el plano de las civilizaciones; la densidad y escala de las actividades económicas, sociales y políticas, que debilita formas territoriales y comunidades cívicas; una geopolítica y regionalización desorganizadora de los sistemas interestatales (Held et al., 2002).

En busca de las singularidades de la globalización, se ha empleado repetidamente la categoría "fragmentación" en estudios sobre grupos sociales, identidades, culturas, cuestiones urbanas, estructuras económicas o laborales, caracterizándola como un hecho destructor de espacios, estructuras sociales o culturas, en perjuicio de una supuesta integración, unidad y armonía previamente existentes. En la perspectiva de Touraine (1997) es significativa la fragmentación de las experiencias de los individuos, y el hecho de que elementos globalizados como flujos financieros, bienes de consumo y medios de comunicación llegan a estar separados de las organizaciones sociales concretas.

Aunque es casi un lugar común asociar fragmentación a situaciones adversas como desigualdad, polarización, riesgo, vulnerabilidad, crisis, exclusión, precarización, segregación, algunos autores, como Giddens (2008), admiten que la globalización conlleva fragmentación tanto como coordinación; y que puede ocurrir una integración de sistemas diferenciados (Luhmann, 1998). Así mismo, se aprecia el influjo de redes globales articulando individuos, sectores económicos, cadenas productivas, agrupaciones de firmas y estrategias productivas (Castells, 1998). En esa dirección, Deffner y Hoerning (2011) defienden que la fragmentación no necesariamente es un límite de los procesos de globalización, sino que más bien puede ser la clave de su dinámica, a condición de que las partes se conecten y potencien en una red. La lógica binaria de incluido-excluido, orden-desorden, que se ha manejado, impide apreciar que, si bien hay desconexiones entre los fragmentos, también son importantes las interconexiones 
e interdependencias que simultáneamente se crean.

Teniendo como trasfondo estas visiones contrastantes de la fragmentación, analizaremos importantes cambios en las esferas institucional, empresarial, urbana y laboral, ocurridos en el sector salud bogotano, sopesandolasinclusionesexclusiones; desconexiones-interdependencias; integraciones-precarizaciones. En el contexto de la actual globalización, mediante políticas públicas y planes productivos, se intenta controlar y gestionar el sector, generándose un escenario heterogéneo en el que el pluralismo estructurado y el lucro privado, como propulsores del sistema, hacen coexistir dispares prácticas. Por ejemplo, las modalidades más regresivas de negocio, al lado de algunos esfuerzos por conformar redes especializadas e interconectadas, armonizadas en la lógica competitiva de los clústeres.

La indagación se apoya en una revisión amplia de documentos públicos y privados (empresariales y gremiales), artículos de prensa y 32 entrevistas semi-estructuradas con actores claves del sistema de salud ${ }^{5}$ en el área metropolitana de Bogotá. Se dialogó con representantes de agremiaciones de la rama de la salud, directivos de entidades, trabajadores de base de la salud, sindicalizados y no sindicalizados, entre otros informantes clave, que se seleccionaron usando un muestreo de bola de nieve. El tema se desarrolla del siguiente modo: comienza con el marco reglamentario que privatizó el sistema de salud colombiano; a continuación, identificamos las principales estrategias empresariales y tendencias en el manejo del sector salud en Bogotá como campo de inversiones; después se describen algunas consecuencias laborales; finalmente, se examinan nuevas prácticas y proyecciones hacia el mercado internacional.

5 Se leyó y solicitó a todos los participantes consentimiento informado, garantizando la confidencialidad de sus datos personales, así como el uso de la información para fines exclusivamente académicos bajo los estándares ESOMAR. No es posible mencionar nombres de entrevistados o las entidades a las que pertenecen dado que se tuvo un compromiso de reserva y confidencialidad, evitando identificar personas u organizaciones.

\section{El sistema de salud colombiano: de la privatización al desfinanciamiento}

Desde la primera mitad del siglo XX, la prestación de servicios de salud en Colombia registra un difuso y lento tránsito de la idea de caridad a la de beneficencia, luego a la de asistencia pública y más tarde a la de sistema de salud como función del Estado, enmarcando esta trayectoria dentro de una lógica de acceso diferencial para cada grupo social (Hernández, 2004). Por otra parte, una conjunción de factores como la limitación de recursos del Estado y su lógica liberal en materia de protección social, la tendencia del empresariado industrial más próspero a establecer para su fuerza de trabajo un restringido sistema patronal de salud y el interés del gremio médico por centrar su práctica profesional independiente en los sectores de más elevados ingresos, condujeron a que en materia de seguridad social se establecieran amplias disparidades regionales, urbano-rurales y sociales. Desde los años 80, y como antesala de las políticas neoliberales que vendría en la siguiente década, se registra el debilitamiento del Seguro Social, que era el máximo logro del Estado dentro de su limitada concepción (Mesa, 2018).

\subsection{La reorganización del sistema de salud tras la ley 100}

La Constitución Política de 1991 introdujo cambios determinantes para la prestación de la salud, al reconocer que Colombia es un Estado Social de Derecho, en el cual la salud se convierte en un derecho universal que pueden reclamar las personas y que debe ser garantizado por el Estado. De ahí, deriva la Ley 100 de 1993 que garantiza la oportunidad y diversidad en el acceso a la salud, crea el Sistema General de Seguridad Social en Salud (SGSSS) y define la responsabilidad que tiene el Estado de coordinarlo y controlarlo, sobre la base del subsidio a la demanda y la meta del 100\% de cobertura (Congreso de la República de Colombia, 1993). Las Empresas Promotoras de Salud (EPS), públicas o privadas, han cobrado importancia por su rol de intermediación financiera de la salud, al administrar los recursos 
económicos destinados a la atención en salud ${ }^{6}$ y contratar directamente con proveedores de servicios de salud (Institutos Prestadores de Servicios de Salud -IPS-), bajo dos regímenes. El primero, el contributivo, determina el acceso de trabajadores y pensionados, con sus respectivas familias a un conjunto estándar de servicios y medicamentos llamado Plan Obligatorio de Salud (POS). El segundo, el subsidiado, es dirigido a sectores de población focalizados por su vulnerabilidad socioeconómica.

La segmentación del aseguramiento se profundiza con la existencia de sectores con planes complementarios, medicina prepagada (para afiliados con alto poder adquisitivo) y modalidades como pólizas internacionales (para trabajadores expatriados) y otros particulares, entre los cuales encontramos los turistas de salud con planes de salud asociados a servicios de hotelería. La Ley 100 de 1993 (Congreso de la República de Colombia, 1993), inspirada en el modelo de "pluralismo estructurado" (Londoño y Frenk, 1997), estableció por lo tanto una nueva relación Estado-mercado, donde la atención médica se ha vuelto un bien privado y se amplió como campo de inversión. Se constituyó de tal manera un sistema con varios escenarios de atención, en el que interactúan aseguradores, prestadores y entidades públicas dentro de un marco de competencia regulada (Hernández, 2002; 2017).

Entre las deficiencias de este modelo, después de más de 20 años de funcionamiento, se puede señalar que no logró resolver el déficit estructural en materia de oferta sanitaria: en 2015, y para 1000 habitantes, el país contaba con 1,8 médicos (contra 3,4, en promedio, para los países de la Organización para la Cooperación y el Desarrollo Económicos (OCDE)); 1,1 enfermeros (contra 9); 1,6 camas de hospital (contra 4,7). Asimismo, la mortalidad infantil (que por

6 Con el propósito de administrar los recursos del SGSSS, el artículo 218 de la Ley 100 de 1993 (Congreso de la República de Colombia, 1993) creó el Fondo de Solidaridad y Garantía del Sector Salud (FOSYGA), a partir del cual se realizan las transferencias de los recursos públicos a las EPS sobre la base de la Unidad de Pago por Capitación (UPC): el valor fijo que se reconoce por cada uno de los afiliados para cubrir las prestaciones del POS. ser mortalidad evitable se considera como un indicador fundamental de calidad del sistema de salud) registra niveles altos: 17,1 muertes de menores de 1 año por 1000 nacidos en Colombia, en 2015, contra -por ejemplo- 14,6 en Brasil, 12,1 en México, 6,9 en Chile y un promedio de 4 para los países de la OCDE (OCDE, 2017). Esta cifra ascendió a 11,5 para Bogotá, en 2016. La cual no deja de ser bastante alta por tratarse Bogotá de una metrópoli con mayor accesibilidad al sistema de salud (DANE, 2018).

\subsection{De las dificultades de acceso a la salud a la quiebra de hospitales}

La puesta en funcionamiento de esta política pública ha mostrado fallas estructurales, en donde, si bien ha existido un crecimiento en el aseguramiento, no se puede decir lo mismo respecto al acceso. El cual aún está lejos de ser garantizado para el $100 \%$ de la población (Agudelo et al., 2011; Ayala, 2014). Para un gran número de afiliados, hay considerables restricciones en sus tratamientos, formulaciones, exámenes diagnósticos y otras prestaciones que están mediadas por dispendiosos trámites. Es también usual la negación de servicios y medicamentos, ante lo cual el afectado solo puede invocar el amparo legal de la tutela. La Corte Constitucional se ha referido al elevado uso de este recurso en derechos de salud, que en 2018 ascendió en todo el país a más de 197.000 tutelas. Lo que significa que el $33 \%$ de la totalidad de tutelas interpuestas para solicitar amparo a derechos esenciales, se refieren a temas de salud. Esto se considera excesivo por todos los analistas (El Tiempo, 2019).

La no cancelación, o dilación injustificada del pago de los servicios por parte de las aseguradoras, es otro de los factores perturbadores del sistema, que afecta seriamente a los prestadores y, para varios sectores de afiliados, repercute en la calidad de la atención. Este entorpecimiento en el flujo de recursos fue estudiado por la Asociación Colombiana de Hospitales y Clínicas (ACHC). Dicho estudio estimó que, en junio de 2017 , las deudas contraídas por las EPS (con una muestra de 153 IPS, sobre un total de 1.800 ) alcanzaron los $\$ 8,2$ billones de pesos 
(aproximadamente $\$ 2,5$ mil millones de dólares). Motivo por el cual, de las 910 Empresas Sociales del Estado (ESE) u hospitales públicos, el $63 \%$ estarían en riesgo fiscal y financiero medio y alto; 32 habían sido liquidadas; 7 habían tenido intervención forzosa; y otras cuantas habían sido fusionadas y privatizadas (ACHC, 2018). Las estadísticas más recientes revelan que, hasta 2016, se habían cerrado más de mil camas de pediatría (350 en Bogotá), afectando directamente el acceso a la salud de los niños (Gossain, 2018).

La afectación de la estructura pública de la salud y de amplios sectores de ciudadanos puede ilustrarse con el caso del Hospital San Juan de Dios (HSJD). Este, otrora principal centro asistencial de Bogotá, atendía pacientes de bajos ingresos y se transformó, en los 60 , de hospital de caridad a complejo universitario. Las condiciones de competencia por los usuarios, bajo el nuevo sistema, lo dejaron en desventaja, causando disminución de pacientes e ingresos. La crisis financiera, que intentó ser mitigada con donaciones de gobiernos extranjeros, ocasionó que, en 2001, la Superintendencia Nacional de Salud decretara la intervención forzosa. La cual fue culminada, en el 2004, por medio de la resolución 1317, que pidió a los aseguradores trasladar a los enfermos hospitalizados en el HSJD y abstenerse de mandar más pacientes a la institución.

Después de varios años de cierre -durante los cuales los trabajadores del HSJD, acompañados de organizaciones sociales, han mantenido su lucha bajo la consigna 'El San Juan de todos'el Tribunal Administrativo de Cundinamarca ordenó, mediante sentencia del 25 de enero de 2018, la reapertura del hospital, preservando su vocación para la atención de personas de bajos recursos. Mientras esto ocurría, los distintos gobiernos distritales, en el periodo del 2000 al 2019, impulsaron una serie de iniciativas urbanas, que buscan hacer del HSJD un polo urbano que impulse el desarrollo inmobiliario y el turismo de salud en el centro de la ciudad.

La teoría de Harvey (2013) sobre la generación de renta monopolística, por medio de especulación sobre el futuro de la tierra o creando unas características únicas sobre la tierra, se verifica acá para explicar este proceso en el que segmentos del sistema de salud entran en crisis. Luego viene la quiebra y el cierre de hospitales, públicos o privados, al mismo tiempo que los mecanismos de privatización del sistema permiten que sectores inversionistas alcancen una posición dominante al adquirirlos. De tal modo, la crisis del sector salud es percibida por los grupos empresariales y fondos de inversión como una gran oportunidad financiera.

\section{Prácticas empresariales en el sector salud: generar ganancias más allá de la prestación del servicio}

Desde el punto de vista de los agentes privados, el sistema de salud colombiano ofrece una muy baja rentabilidad para la prestación de servicios de salud dentro del marco institucional de los regímenes subsidiado y contributivo (Jaimes y Rincón, 2009). En un contexto de déficit estructural de la provisión en salud, las prácticas empresariales son entonces claves para entender cuáles son las lógicas y dinámicas dentro de las que se estructura el negocio de la salud.

\subsection{Lógicas de integración vertical}

Una de las tendencias significativas como estrategia y práctica de negocio en el sector salud, en Bogotá, ha sido la formación de conglomerados, convertidos en casa matriz a partir de la razón social de una IPS, clínica o empresa de medicina prepagada e, incluso, de una agremiación de especialistas. Las IPS más grandes en el país crearon su propia EPS de régimen contributivo tras la ley $100 \mathrm{y}$, desde entonces, construyen, compran o integran clínicas, proveedores de todo tipo y hasta instituciones educativas, escuelas de auxiliares y laboratorios.

En este sentido, son notables tendencias a la integración vertical que buscan generar ganancias a partir de las actividades que no son de la prestación del servicio de salud (aseguramiento, insumo, capacitación, transporte) y a la acumulación de los recursos recibidos por la Unidad de Pago por Capitación (UPC), a través de la 
conformación de un circuito financiero (Jaimes y Rincón, 2009; Hernández, 2002).

Lo anterior, a pesar de que "su naturaleza de aseguradoras les exige una administración del riesgo financiero" (Jaimes y Rincón, 2009, p. 92). Así, entre las 100 empresas más grandes del país en 2017, por sus ingresos operacionales, encontramos 13 EPS. Siendo las más grandes con ingresos operacionales, superiores a $\$ 1,7$ billones de pesos, en respectivo orden: la Nueva EPS, Coomeva (que actualmente posee su propia entidad bancaria: Bancoomeva'), Sura, Sanitas, SaludTotal y Medimás (Semana, 2018). Además, diversas organizaciones, que poseen EPS en el régimen contributivo, ofrecen también medicina prepagada, planes complementarios y pólizas de salud. Situación por demás polémica por el poder de mercado que les brinda a estas empresas y por la posibilidad de exigir, al usuario del plan adicional, afiliarse antes a alguna EPS del régimen contributivo (Jaimes y Rincón, 2009).

Aunque la Ley 1122 de 2007 limitó la integración vertical de las EPS a un $30 \%$, como máximo, esta práctica se ha seguido evidenciando como una tendencia fuerte en algunos grupos empresariales o conglomerados del sector salud (Congreso de la República de Colombia, 2007). Ahora bien, es preciso recordar que, si bien la mayoría de las EPS son privadas, una parte importante de los hospitales y el personal que prestan los servicios a las EPS son públicos. Muchos, debido a las incoherencias del sistema, permanecen al borde de la quiebra: los prestatarios públicos del servicio de salud se encuentran en una posición muy vulnerable frente a los aseguradores privados. Estos últimos realizan altas ganancias, gracias a una gestión

7 En 2016, tras una operación de aproximadamente 50 millones de dólares, Christus Health adquirió el 50\% de las acciones de Sinergia Salud, una de las compañías de Coomeva propietaria de las clínicas Farallones de Cali, Palma Real de Palmira, Somer de Medellín y el servicio Hospital en Casa. La compañía estadounidense obtuvo participación en la junta directiva, gerencia y administración de Sinergia Salud. La alianza comprendió, además, el $10 \%$ de Coomeva Medicina Prepagada -con la posibilidad de ampliarlo hasta el $45 \%$-, así como el $5 \%$ de Coomeva EPS. no regulada de los recursos públicos que les llegan vía el pago de la UPC.

\subsubsection{Apertura a la participación del capital privado global y nuevas modalidades de integración vertical}

A pesar de las conocidas debilidades y fallos del sistema de salud en su función social central, tanto en la esfera de las políticas públicas como en los planes gremiales y corporativos, se percibe en los últimos años la búsqueda de nuevos negocios, inversiones extranjeras o de naturaleza global. Con ese propósito, se dinamizó el régimen de zonas francas en el país (Ley 1004 de 2005 -Congreso de la República de Colombia, 2005-), lográndose un notable crecimiento, hasta alcanzar 105 en total, que incluyen 13 zonas francas en salud. Cifra que sitúa a Colombia como el país de América Latina con mayor cantidad.

En 2016, la entrada en vigencia del decreto 2147 de 2016 transformó el régimen de zonas francas, para hacer más ágil su aprobación y estimular el desarrollo de actividades avanzadas tecnológicamente y con proyección internacional. Esta norma precisa aspectos de las Zonas Francas Permanentes Especiales (ZFPE), de tipo industrial o de servicios -también conocidas como uniempresariales- en la que una única firma, que cumpla ciertos requisitos de inversión y mínima generación de empleo, sin importar su localización, puede contar con una serie de ventajas y trato excepcional en materia aduanera, tributaria y de comercio exterior (Ministerio de Comercio, Industria y Turismo, 2016).

A diferencia del caso de Dubai (Ganji, 2015) y de otras experiencias de zonas francas de salud en países emergentes como Turquía (Deloitte, 2014), Colombia es el único país en el mundo donde se han creado zonas francas con carácter uniempresarial. En ese sentido, las compañías únicas y sin obligación de localizarse en un espacio específico, adquieren beneficios tributarios e incentivos económicos. De modo que, lo que en otros países se hace para conseguir proximidad, integración horizontal de firmas, 
servicios compartidos, diversificación y cooperación de actores, como estrategias de innovación y competitividad, en Colombia se reduce a un mecanismo para atraer inversiones dentro de una lógica de integración vertical en torno a firmas aisladas, beneficiándose a los más grandes grupos del sector o a grupos financieros de orden global (ver Tabla 1). Este tipo de negocio está destinado a pacientes con alto poder adquisitivo, atiende de manera exclusiva a pacientes particulares, con pólizas, medicina prepagada o planes complementarios. Como es el caso de la zona franca Los Nogales, en el norte de Bogotá, en la cual el área de hospitalización cuenta con estándares de hotelería de lujo.

Las zonas francas se proyectan para recibir turistas de salud y no constituyen una herramienta reglamentaria que busca mejorar la prestación de servicios y el derecho a la salud de los colombianos. Si bien las Zonas Francas han mostrado decaimiento en los últimos años, como campo de inversión, una modalidad más propicia a la interacción entre firmas, conocida como Alianzas Público-Privadas (APP), ha crecido en el sector salud desde 2014, con la conformación de alianzas de grupos nacionales e

Tabla 1. Zonas Francas de Salud aprobadas en Colombia

\begin{tabular}{|c|c|c|c|}
\hline Nombre & $\begin{array}{c}\text { Fecha de } \\
\text { aprobación }\end{array}$ & Localización & Inversionistas identificados \\
\hline $\begin{array}{l}\text { Centros Especializados de San } \\
\text { Vicente Fundación }\end{array}$ & 2009 & Rionegro (Antioquia) & San Vicente Fundación (Colombia) \\
\hline Clínica Portoazul & 2009 & $\begin{array}{l}\text { Barranquilla } \\
\text { (Atlántico) }\end{array}$ & United Health Group (Estados Unidos) \\
\hline Clínica Los Nogales & 2009 & Bogotá D. C. & Carlos Ardila Lülle (Colombia) \\
\hline Fundación FOSUNAB & 2010 & $\begin{array}{l}\text { Floridablanca } \\
\text { (Santander) }\end{array}$ & Carlos Ardila Lülle (Colombia) \\
\hline Clínica Hispanoamérica S.A.S. & 2010 & Pasto (Nariño) & $\begin{array}{c}\text { Roberto Cocheteux, Joseba Grajales, Iñigo } \\
\text { Gómez (España) }\end{array}$ \\
\hline $\begin{array}{l}\text { Fundación Cardiovascular de } \\
\text { Colombia S.A.S.A partir de 2016: } \\
\text { Hospital Internacional de Colombia }\end{array}$ & 2012 & $\begin{array}{l}\text { Piedecuesta } \\
\text { (Santander) }\end{array}$ & $\begin{array}{c}\text { Carlos Ardila Lülle (Colombia). A partir de } \\
\text { 2016: Rizk Ventures y Goldman Sachs } \\
\text { (Estados Unidos). Crédito con el Banco } \\
\text { Mundial. }\end{array}$ \\
\hline $\begin{array}{l}\text { Sociedad Clínica Cardiovascular } \\
\text { Corazón Joven S.A. }\end{array}$ & 2012 & Neiva (Huila) & \\
\hline Medical Duarte Z.F. S.A.S. & 2012 & $\begin{array}{l}\text { Cúcuta (Norte de } \\
\text { Santander) }\end{array}$ & \\
\hline $\begin{array}{c}\text { Fresenius Medical Care Servicio } \\
\text { Renal S.A.S. }\end{array}$ & 2013 & $\begin{array}{l}\text { Cúcuta (Norte de } \\
\text { Santander) }\end{array}$ & Fresenius Medical Care (Alemania) \\
\hline $\begin{array}{c}\text { Centro Hospitalario Serena Del Mar } \\
\text { S.A. }\end{array}$ & 2014 & Cartagena (Bolívar) & $\begin{array}{l}\text { Fundación Santafé - Bogotá (Colombia). } \\
\text { Crédito con el Banco Mundial y el Banco } \\
\text { Interamericano de Desarrollo (BID) }\end{array}$ \\
\hline $\begin{array}{c}\text { Clínica de Marly Jorge Cavelier } \\
\text { Gaviria }\end{array}$ & 2016 & Chía (Cundinamarca) & $\begin{array}{c}\text { Clínica de Marly Santafé - Bogotá (Colom- } \\
\text { bia) }\end{array}$ \\
\hline $\begin{array}{l}\text { Clínica Universitaria Medicina } \\
\text { Integral S.A.S. }\end{array}$ & 2016 & Montería (Córdoba) & \\
\hline Diacor Soacha Zona Franca S.A.S. & 2016 & $\begin{array}{c}\text { Soacha } \\
\text { (Cundinamarca) }\end{array}$ & Ashmore Investment Group (Reino Unido) \\
\hline
\end{tabular}

Fuente: Ministerio de Comercio, Industria y Turismo (2018) y datos propios. 
internacionales. El impulso lo dio el gobierno de Juan Manuel Santos (2014-2018), al poner en marcha la política de las Redes Integradas de Servicios de Salud (RISS), donde las empresas privadas, de cualquier tamaño, que hagan parte de la cadena de producción de la salud, puedan celebrar convenios que les permita prestar los servicios de manera complementaria.

\subsubsection{Cuando las empresas de salud ingresan a los negocios inmobiliarios y transforman el espacio urbano}

Además de la propensión a la integración vertical, los grandes grupos empresariales en salud han incursionado en los negocios inmobiliarios, bajo diversas modalidades. Según las estadísticas del DANE (2016), la expedición de licencias para la construcción de hospitales se ubicó, en el $\mathbf{2 0 1 5}$, en $\mathbf{5 6 7 . 0 7 4}$ metros cuadrados, con un crecimiento de $32,9 \%$. En cambio, en 2014 , totalizó 426.587 metros, lo cual demuestra que los activos inmobiliarios de la salud se vuelven un atractivo en sí mismo para los inversionistas (El Tiempo, 2016).

La Clínica San Rafael es un caso ilustrativo. Se trata de una institución privada católica fundada en 1929 y cuyo patrimonio se vio afectado, desde el 2012, por el no pago de los servicios prestados a las EPS. Tal como lo reconoció Carlos Vicente Rada, director de la Orden Hospitalaria San Juan de Dios, organismo que era entonces propietario de la Clínica San Rafael (Cantillo, 2012). A raíz de esta situación, la entidad emprendió un proceso de despidos de trabajadores y después fue vendida a la multinacional Tribeca. Esta compañía, que pertenece al grupo Rizk Ventures, opera en Colombia como "National Clinics" y cuenta con la Clínica los Nevados (Pereira), el Hospital Internacional de Colombia (Santander) y la National Clinic (Bogotá). Tribeca introdujo un modelo de negocio que separa la prestación de servicios de salud del negocio inmobiliario: administra los servicios hospitalarios y un joint-venture, del cual hace parte el grupo Goldman Sachs, que maneja la finca raíz (Dinero, 2016). De tal manera, las pérdidas del primero no pueden repercutir en el segundo.
Dentro de una estrategia de negocio diferente, la clínica Country se enfoca en la prestación de servicios de salud de alta gama para particulares nacionales, medicina prepagada y turismo de salud. Todo articulado, por una parte, a su internacionalización y, por otra, al negocio inmobiliario. Luego de ser adquirido por la holding chilena Banmédica, en 2007, el Grupo Empresarial Country (que reúne a las clínicas Country y La Colina en Bogotá, la Zona Franca en salud Portoazul, en Barraquilla, y la aseguradora Colmédica Medicina Prepagada) fue comprado, en 2018, por la compañía estadounidense de seguros médicos United Health Group, por un valor cercano a los $\$ 2.800$ millones de dólares (El Tiempo, 2017). Estas ventas hacen parte de un proceso de adaptación de la institución a los requerimientos internacionales del turismo en salud y posibilitaron la consolidación de la infraestructura hospitalaria. Asimismo, es destacable el caso de la clínica Country, en Bogotá, por su contribución a la especialización económica y transformación radical de los espacios urbanos de su entorno; lo anterior con apoyo de programas urbanísticos promovidos desde el gobierno distrital de Bogotá, como el Plan de Implantación, mediante el cual se aprueba la construcción de grandes superficies de escala metropolitana.

En esa dirección, la resolución 0940 de junio de 2018, de la Secretaría Distrital de Planeación, autorizó la intervención de un área de $3630 \mathrm{~m}^{2}$ en pleno corazón financiero y económico de Bogotá, aplicando un esquema en que la enajenación voluntaria de los predios requeridos, para la ampliación de la clínica, se compensa con un porcentaje equivalente en la nueva construcción. De esta forma, el grupo empresarial alcanza el control sobre el suelo urbano y se beneficia con altas plusvalías en un sector altamente densificado y desarrollado. Caso similar es la Clínica Portoazul, en Barranquilla, que se ha beneficiado de la reglamentación de zona franca uniempresarial en salud, ubicándose en el sector de expansión urbana más dinámico de la metrópoli costera, lo cual le permite mantener una alta valorización como activo inmobiliario. Este mismo proceso se observa alrededor de las zonas francas de salud, ubicadas en las 
áreas de más alta valorización de las periferias metropolitanas: clínica Marly en Chía y clínica Serena del Mar en Cartagena. Todas articuladas a nuevos frentes de urbanización residencial de standing, asociados con la construcción de centros comerciales de última generación y de nuevas sedes de instituciones de educación superior privadas prestigiosas.

La concentración de infraestructura en manos de grandes empresas nacionales y extranjeras valoriza rápidamente los activos inmobiliarios, en particular para los consultorios médicos, como ocurre en el entorno de la Clínica el Country. Esta dinámica inmobiliaria de la salud lleva al surgimiento de nuevas formas de transacciones, como el alquiler de consultorios médicos, odontológicos y psicológicos por horas, el cual se ha vuelto el nicho de negocio aprovechado por la plataforma Tueme. El proceso del Hospital San Juan de Dios es también demostrativo de otras implicaciones para la producción de ciudad. Una vez consumada su quiebra, las administraciones distritales ligaron su reapertura a un proyecto urbano e inmobiliario que incluye la renovación urbana de los barrios circundantes, con construcción de viviendas en Las Cruces y San Bernardo.

El Plan de Ordenamiento Territorial de Bogotá (POT) determinó en 2004 que, en el área en que se encuentra el HSJD, se debía edificar un centro hospitalario que promoviera la prestación de servicios a usuarios nacionales y extranjeros, fomentando el turismo en salud y los servicios complementarios que se derivan. Lo anterior se materializó, en el 2007, al expedirse el decreto 492 que puso en operación el Plan Zonal del Centro (PZCB), que incluyó, como uno de sus espacios estratégicos de borde, el HSJD y las instituciones circundantes. Este ambicioso plan de remodelación e inversión para el HSDI, conocido como "Ciudad Salud", consta de un proyecto de renovación urbana para la zona hospitalaria, la creación de una serie de infraestructuras para servicios complementarios y el apalancamiento de proyectos urbanos en su entorno, que faciliten la reinversión en el centro, en particular el Plan Parcial de Renovación Urbana "San Bernardo Tercer Milenio".
En 2011, el Plan Maestro de Turismo, en Bogotá, reiteró que este proyecto debía estar orientado al turismo en salud, por lo que se propuso la construcción de hoteles, superficies comerciales y otros tipos de negocios que satisficieran las demandas de los nuevos usuarios. El desarrollo de este megaproyecto urbano con inclusión del HSJD, fue orientado hacia un modelo de inversión APP. Para el cual, la firma Currie \& Brown -especialista en la materia- estableció que el proyecto sería una APP de Bata Gris-Verde, que incluya el diseño, construcción, operación y mantenimiento de la infraestructura; mantenimiento de los equipos biomédicos y la operación de los servicios complementarios como el aseo, la vigilancia, la alimentación, la lavandería, entre otros (Currie y Brown, 2018). Este complejo hospitalario funcionará como una Red Integrada de Servicios de Salud (RISS), que unifique a las localidades de Santa Fe, Candelaria, Mártires y San Cristóbal, sumando en total 62 instituciones de diferente tamaño y vocación, destinadas a conformar la "red centro oriente". De tal manera, el gobierno distrital fijó el desarrollo de su infraestructura pública por medio de APP, para así entregar su ejecución y administración a multinacionales. Así lo dejó saber la administración distrital en el Segundo Foro Internacional de Asociaciones Público-Privadas en Salud para Bogotá, realizado en 2018.

En síntesis, el caso Country, ubicado en la zona norte de la ciudad, dentro de los entornos más costosos y privilegiados; y el caso HSJD, en un sector céntrico, popular y pauperizado urbanísticamente, han sido objeto de políticas similares. En ambos casos, aunque con más adelanto en el primero, están en marcha instrumentos de planificación urbana destinados a generar suelo, de origen privado (caso Country) o público (HSJD), para el beneficio de los grupos empresariales que, con la venia gubernamental y los recursos públicos, derivan utilidades injustificadamente elevadas de estos negocios inmobiliarios asociados a la salud. En un contexto de déficit estructural de la oferta, la única contrapartida para la colectividad es una probable mejora en la prestación del servicio de salud. 


\subsection{Lógicas de explotación laboral}

Las tendencias de fragmentación, segmentación y polarización perceptibles en los mercados laborales bajo las dinámicas globales (Durand, 2011), exploradas para el sector salud colombiano, en medio de su complejidad, evidencian la consolidación de ámbitos heterogéneos de manejo laboral, puesto que reproducen la diversidad de condiciones institucionales y económicas que se han descrito. Desde la observación empírica, se advierte que, aunque existe un núcleo de clínicas y entidades en donde se han mantenido e incluso mejorado estándares laborales, predominan en la mayoría de los casos prácticas que, de modo diferenciado, erosionan estándares de protección social y garantías laborales, ocasionando la ruptura y disolución de estructuras ocupacionales. Se trata también de otro orden de estrategias de acumulación promovido por las EPS, que reduce costos mediante mecanismos de explotación laboral, vía externalización, tercerización y precarización del personal de salud.

Desde que la ley 100 externalizó los servicios hacia las EPS, empezó una cadena de externalizaciones: EPS subcontratando, mediante convenios, con IPS privadas y estas, a su vez, externalizando áreas misionales y auxiliares de servicio. Además, basadas en su creciente poder de mercado como conglomerados de la salud, indirectamente ocasionan precarización laboral en las clínicas que no son de su propiedad, con el retraso o no pago de los servicios prestados (ACHC, 2015). Las EPS con artimañas para retener pagos (auditorías, glosas, re-facturaciones y renegociación de pasivos) acaparan recursos públicos y para-fiscales de la salud (Cadavid et al., 2016). Más aún, la legislación responsabiliza a los hospitales por la auditoría de las glosas, obligándolos a gastar más en personal administrativo (Quintana et al., 2016). Esta situación agudiza el déficit de los hospitales, llevando no solo a situaciones de mora con proveedores, sino también con empleados, y a diversas afectaciones laborales (ACHC, 2015).

Las EPS, como administradoras financieras del sistema de salud, también están tercerizando personal o servicios médicos, cerrando y externalizando áreas de servicios, inicialmente con el uso de Cooperativas de Trabajo Asociado (CTA) -hasta su reciente prohibición- y después, mediante una variada gama de modalidades, como empresas de servicios temporales, ordenes de servicios y contratos sindicales (Aricapa, 2008; Puig y Osorio, 2014). La subcontratación también persiste como tercerización naturalizada, donde la empresa matriz subcontrata servicios de su core bussiness a otra secundaria (Puig y Osorio, 2014). Se tercerizan labores como aseo, mensajería, cafeterías, lavandería, pero también las labores de enfermeras supernumerarias, por ejemplo, en Nuestra IPS, en el Hospital San Ignacio y en la Clínica Reina Sofía. En esta última, llama la atención una modalidad de outsourcing de servicios, donde la clínica transfiere la actividad a empresas subcontratistas creadas por las jefes de enfermería de la misma, que se turnan la prestación del servicio en un piso de medicina prepagada.

Se ha hecho usual la coexistencia en un mismo espacio de empleados directos y tercerizados bajo diferentes modalidades, con disímiles salarios y precariedades. Así se aprecia en la Clínica Marly, en donde a partir de la flexibilización laboral introducida con la Ley 50 de 1990 (Congreso de la República de Colombia, 1990), se empezó a contratar personal con empresas proveedoras de temporales. En 2013, el sindicato Sintramarly denunció, ante el Ministerio del Trabajo, esa tercerización de personal misional. El proceso de investigación duró dos años, hasta que el Ministerio expidió una sanción tanto para la Clínica Marly como para "Ocupar", la empresa de servicios temporales que más trabajadores concentraba en la clínica en ese momento. La clínica fue obligada a contratar directa y formalmente a 400 trabajadores, a cambio de que se le levantara la sanción. No obstante, Marly no les concedió nivelación salarial y los vinculó con un convenio en condiciones inferiores a las de los trabajadores de planta originales.

En general, las clínicas y hospitales recurren a trabajadores independientes o monotributistas a los que imponen contratos de prestación de servicios como supernumerarios y copropietarios de empresa temporal, para así aplicarles 
cláusulas contractuales glosando servicios facturados por obras o servicios. Con lo anterior se oculta lo que en los hechos es una relación laboral normal, en sus aspectos de subordinación y cumplimiento de labores misionales de la entidad. También son usuales contratos con alta diferencia entre salario básico y total, y uso de bonos sodexo-pass ${ }^{8}$ para reducir la base sobre la que se calculan las prestaciones sociales. Todas estas prácticas atípicas de contratación en el sector configuran una precarización multidimensional de un segmento de trabajadores de la salud indeterminado aún.

Cierta polarización de condiciones y segmentación es notoria en niveles salariales contrastantes entre profesiones médicas y paramédicas: enfermeras especializadas y no especializadas; y entre estas y médicos; del mismo modo entre médicos generales y especialistas (Bohórquez, 2017). Con lo cual se consolidan en el sector, y aún a nivel de clínicas, segmentos laborales agudamente diferenciados, dependiendo del grado de externalización y tercerización de los vínculos laborales, que varía según las lógicas rentistas presentes en las clínicas y el ámbito de negocio de cada entidad. En algunas clínicas y entidades, las deterioradas condiciones del sector de trabajadores tercerizados se disimulan resaltando las buenas condiciones de los profesionales de planta, como una fuente de valor agregado. Publicitándolas, por ejemplo, ante potenciales pacientes internacionales.

Lo más destacable, respecto al tema laboral, es que se han generado diversas modalidades de precarización del trabajo y deterioro de la situación profesional, prácticas que en la medida en que hacen parte de escenarios de corrupción y acciones fraudulentas contra el sistema, serían otra forma indebida de extracción de beneficios, que en muchos casos se presenta en combinación con las trasgresiones y conductas

8 Bonos para adquirir productos de consumo final, que subsidia una empresa a sus empleados a través de la empresa Sodexo, como parte del pago de su salario bajo este modo de cheque canjeable en puntos con convenio, o como incentivo emocional, adicional al salario. más típicamente rentísticas. La diversidad de estructuras y procesos en el sistema, en el que se pretende dar espacio a la intervención reguladora del Estado, tanto como a la competencia entre inversionistas privados, indudablemente ha creado un ambiente propicio al rentismo. De acuerdo a Tullock (2005), este es un fenómeno de múltiples facetas, en el que los actores empresariales emplean conexiones políticas para obtener ventajas en la actividad económica. Del mismo modo, el ejercicio de poderes monopólicos y el aprovechamiento de deficiencias en el control público, para obtener una cuota excesiva de ganancias, es acudiendo al lobby de regulaciones económicas, la captura del ente regulatorio, la colusión entre firmas y agencias gubernamentales.

Es característico, en el sistema colombiano, el fraude a las condiciones de transparencia, libre concurrencia y competencia en el mercado, integraciones verticales, abuso de posición dominante, apropiación de recursos públicos. Todo lo anterior configura una gama de conductas, que van desde las leves infracciones hasta la abierta trasgresión de normas y el ilícito. Tales prácticas rentísticas, en muchas ocasiones, se acompañan de manejos laborales generadores de deterioro en la calidad de los empleos, y explican la formación, en los escenarios sociales hospitalarios, de manifestaciones específicamente sectoriales de precariedad laboral. Por ejemplo, la incrementada agresión hacia el personal de la salud por parte de usuarios insatisfechos.

\section{Clúster y turismo de salud como potenciales vías hacia la globalización}

Una faceta importante dentro de los cambios económicos en la mundialización de las economías ha sido la conformación y el desarrollo de clústeres. Son muy variados los interrogantes y temas planteados, así como las perspectivas desde las que se ha abordado en las diferentes disciplinas relacionadas con lo territorial y lo urbano, la gestión de organizaciones, negocios, ciencia y tecnología (Navarro, 2003; Hervas-Oliver et al., 2015). 


\subsection{El clúster de la salud: lo urgente no deja ver lo importante}

En Colombia, con el 'cambio de modelo' de los 90, se dejó de lado la intervención del Estado y énfasis en el mercado interno, pero se mantuvo un rol orientador en los organismos estatales, que desde entonces han impulsado diferentes planes para fomentar condiciones de competitividad y productividad a través de acuerdos sectoriales, optimización de cadenas productivas, zonas francas, acuerdos público-privados y clústeres, entre otros. Siguiendo planteamientos de Porter (1991) sobre generación de capacidad competitiva, se buscó aprovechar la proximidad entre clientes y proveedores, así como las externalidades ligadas a la aglomeración y densificación de firmas, especialización, eficiencia en la cadena productiva y capacidad innovadora colectiva.

Es así como varios sectores productivos, entre 1993 y 1994, tuvieron asesoría de la consultora "Monitor" del mismo Porter (Ministerio de Comercio, Industria y Turismo, 2009). Desde estas épocas, ha habido interés por los fenómenos relativos a redes, cadenas, distritos y clúster. La influencia de instituciones supranacionales, como el Banco Interamericano de Desarrollo (BID), el Banco de Desarrollo de América Latina (CAF), del mismo modo que el interés por vincular el país a la OCDE, han motivado aproximaciones a teorizaciones sobre clúster. Entre ellas, las de la Nueva Geografía Económica (Sabel et al., 2012, p. 11) y de la economía de la innovación, que son adoptadas en algunas políticas y gremios colombianos; por ejemplo, políticas de regiones competitivas, áreas metropolitanas y ciudad-región.

En este contexto de políticas, se ubica la "Iniciativa Clúster Salud", liderada desde el 2017 por la Cámara de Comercio de Bogotá (CCB), con la participación de importantes entidades públicas y privadas de la ciudad?: el Servicio Nacional de Aprendizaje (SENA), el Ministerio de Trabajo, el Ministerio de Educación, el Instituto Colombiano

9 La información sobre esta experiencia organizativa se obtuvo principalmente de entrevistas a funcionarios de entidades del clúster -situados en distintos niveles- y a conocedores del sector. de Normas Técnicas (ICONTEC), la Secretaría Distrital de Planeación y la Secretaría de Salud. Entre las privadas, se encuentran entidades productoras de insumos, medicamentos y equipos, consultores especializados en calidad, EPS e IPS (Hospital San José, Clínica de la Mujer, Clínica Marly, Clínica Sabana, Fundación Shaio, Fundación Santafé y Clínica del Country). El clúster está organizado en torno a una junta directiva integrada por líderes de las principales clínicas, un consejo ampliado y mesas temáticas: "calidad", "talento humano", "innovación, investigación y desarrollo", "mercadeo", "infraestructura y entorno". De modo general, esta organización se aprecia como espacio para examinar problemas y buscar soluciones articuladas. Otras tareas de interés son el monitoreo de datos oficiales producidos por observatorios, la generación de parámetros y soporte a las entidades en metrología, la promoción y difusión de eventos, proyectos, congresos, cursos.

La combinación del esfuerzo en las mesas de "calidad" e "infraestructura" facilita ejercicios de benchmarking con las demás entidades, interactuando con la cadena sectorial y con los pares. Aunque la iniciativa de la CCB, con menos de dos años de desarrollo, requeriría más tiempo para mostrar avances, sí es posible señalar algunas tendencias en su implementación, como la prioridad en los temas de infraestructuras y habilitación, y la cuestión de la confianza entre los miembros. El problema de la infraestructura se relaciona con la necesidad que tienen las clínicas y hospitales -entre los cuales hay algunos de cierta antigüedad- de construir nuevas instalaciones, ampliar o adaptar las existentes. Para tal propósito, las clínicas deben acogerse a las directrices del POT, y otras normas urbanísticas y técnicas de la Secretaría de Salud.

Las tensiones generadas en estos temas y la lentitud de los procesos de aprobación de proyectos, han llevado a que los integrantes del clúster analicen conjuntamente estas condiciones. Un primer producto de esta acción colectiva fue un documento con expectativas respecto a temas urbanos, para ser sustentado ante el Consejo de Bogotá. Acciones similares tendientes a expresar puntos de vista ante las autoridades distritales, que consideran que hay desequilibrios por excesiva concentración de 
instalaciones de salud en zonas de la ciudad como las del norte, se adelantan en otros medios gremiales como la ACHC y la mesa sectorial de salud de la Asociación Nacional de Empresarios de Colombia (ANDI).

Por lo anterior, algunos partícipes del clúster consideran que estos esfuerzos corresponden más a urgencias y necesidades inmediatas, que aún no reflejan avances en el sentido de la concentración espacial e interacción productiva propia del clúster. Además de esta integración de esfuerzos, casi cercana a los usuales mecanismos de cabildeo, se advierten iniciativas particulares de algunas IPS para resolver la necesidad de nuevos espacios: el proyecto de ampliación de la Clínica del Country ya mencionado, los ensanches de la Fundación Santafé y de la Clínica de Occidente.

La obligación de acoger normas y estándares de habilitación exigidos por el Ministerio y la Secretaría Distrital de Salud, también ha estimulado la actividad concertada sobre temas como calidad y metrología. Dado que la aplicación de una parte de las normas resultaría tan problemática que podría hacer inviables algunas entidades, o imponer serias modificaciones a sus procesos, se ha valorado la interacción entre entidades reguladoras y prestadoras para crear estándares y ajustes a actividades y condiciones específicas. Considerando la esfera de las mesas de trabajo, de acuerdo a la percepción de sus protagonistas, se nota una falta de compromiso y persistencia en las tareas. Además de insuficiente convicción en la alta dirección de las entidades respecto al proyecto clúster en general. Ilustrativo de esta situación fue lo ocurrido cuando se inició un estudio de costos y calidad, para el cual se debía generar una información que la CCB reuniría, pero los pocos datos aportados resultaron insuficientes para sistematizar. Lo anterior puso de presente la falta de confianza por compartir información entre entidades, como elemento de fondo adverso a la dinámica del clúster.

Respecto a las condiciones y requerimientos para la construcción de un clúster, el examen de algunos indicadores a nivel sectorial tampoco muestra un panorama favorable. En lo tocante a los nexos entre clínicas y otras entidades para realizar investigación, un estudio demuestra poca incidencia de las instituciones en los logros de investigadores académicos y científicos, falta de integración de los modelos organizacionales del hospital y la academia para la investigación, y reducida presencia de entidades del sector público (Jaramillo-Salazar et al., 2008).

En estos procesos del sector salud, que deberían contribuir tanto a la producción de ciudad, como a la construcción de tejido económico, la persistencia de la fragmentación y extrema desigualdad de las firmas y entidades convocadas conforman un escenario en el que políticas económicas e iniciativas gremiales, muy débilmente, esbozan propósitos de ordenamiento para la competitividad y de concertación para alcanzar los plausibles objetivos de optimizar cadenas productivas y promover clúster. Por todo lo anterior, los esfuerzos innovadores se concentran en un reducido número de entidades e inevitablemente reproducen las lógicas de la firma aislada en su integración vertical, desarrollo de portafolios de negocios internacionales, certificaciones de calidad y otras acreditaciones de firmas específicas, que generan algunas concentraciones de infraestructura e interacciones en red con otros establecimientos, pero no una articulación que lleve a verdaderas capacidades competitivas colectivas.

\subsection{Turismo de salud apenas una posibilidad}

Teniendo como referente los logros de países diversos y algunos avances nacionales, se ha planteado la viabilidad de vender servicios médicos, incorporados en planes turísticos y hoteleros, a visitantes extranjeros que llegarían en volumen atraídos por costos más reducidos que en sus países, o que no se prestan allí. Según promotores de entidades gubernamentales y empresarios, el atractivo del bajo costo, sumado a la especialización, imagen de confiabilidad y la óptima gestión de un proceso que combina aportes de clínicas, agencias de turismo y hoteles, conformaría un clúster favorable al desarrollo científico, empleo e ingresos para el país. En la 
expectativa de favorecer un incremento y mayor proyección de la exportación de servicios de salud, se ha creado un marco institucional y de políticas públicas, así como planes de gremios como la CCB (Cámara de Comercio de Bogotá) y la ANDI (Asociación Nacional de Industriales). El CONPES (Consejo Nacional de Política Económica y Social) 3678 de 2010, como Plan Estratégico pensado para el desarrollo del turismo de Salud, definió un modelo sectorial que promueve los acuerdos bilaterales para aumentar el ingreso de pacientes extranjeros y el crecimiento de las inversiones, contando con el establecimiento de las zonas francas en salud, la promoción del bilingüismo, los Programas de Transformación Productiva, la certificación de instituciones dentro del modelo ISQua-ICONTEC (Instituto Colombiano de Normas Técnicas y Certificación).

La factibilidad de estos planes se sustenta en logros como el posicionamiento de cinco clínicas colombianas en escalafones internacionales, y varias alianzas y convenios de ellas con prestigiosos centros mundiales. Ante las posibilidades del turismo de salud, el clúster de la CCB ha hecho análisis del valor agregado posible de alcanzar y ha estudiado experiencias en otros países. Considerando que los logros en materia de complementariedades, sinergias y eficiencia colectiva, en que están comprometidos, son prometedores en relación con la demanda de pacientes extranjeros que buscan no tanto entidades aisladas, sino un sector sólido, con una amplia red integrada y con un agregado amplio de ofertas. Asimismo se valora lo que se ve como fuente de ingresos, lo cual podría resolver la falta de liquidez de las entidades ante los atrasos en pagos que mantienen las EPS.

Sin embargo, aún el desarrollo del turismo de salud no está dentro de los propósitos explícitos del clúster salud de Bogotá. En el contexto nacional, un conjunto de hospitales y clínicas muy destacadas, algunas de las cuales pertenecen al clúster de la $\mathrm{CCB}$, hace varios años atienden pacientes extranjeros, registrándose a nivel sectorial incrementos en este rubro. Se han dado pasos para crear áreas especializadas; lograr las certificaciones y nexos necesarios; promover el bilingüismo y otras calificaciones. Pero por ahora, a la luz de las cifras, el turismo médico en Colombia es un sector apenas incipiente en la economía nacional, ya que se estima que solo el $3 \%$ del total de turistas internacionales, que ingresa al país, lo hace por razones médicas (De la Puente, 2018).

\section{Conclusiones}

Desde los años 90, se ponen en práctica diferentes políticas dirigidas a promover la capacidad competitiva en varios sectores de la economía. Se suceden y, a veces, coexisten diferentes estrategias, como acuerdos sectoriales, coordinación de cadenas productivas, zonas francas, alianzas público-privadas, clústeres. En el sistema de salud, marcado por las nuevas condiciones que crea la ley 100, se profundiza una fragmentación en la que se crean nuevas oportunidades de negocio y extracción de rentas; impactos espaciales y urbanos; población usuaria y políticas laborales. Algunas clínicas y entidades más dinámicas, pertenecientes al sector con mayor capacidad científica y organizativa, en los últimos años han comenzado a hacer aproximación a las estrategias de clúster y nuevas actividades como el turismo de salud, que están siendo promovidas por entidades públicas y gremios privados. Aunque se encuentran en etapas iniciales, y aún no hacen grandes apuestas en el tema, estos actores empresariales muestran disposición a estructurar eficientes intercambios y nexos productivos, generar procesos de localización y concentración de firmas perceptibles en los espacios urbanos.

Sin embargo, el sentido y orientación de estas prácticas parece estar más ligado a tradicionales tendencias de integración vertical, incursión en nuevos negocios que requieren certificación y afinación de estándares, que a las nuevas dinámicas de interacción de clúster, destinadas a construir relaciones de confianza, innovación y competitividad. Además, a pesar de algunos ajustes normativos, no contribuyen a mejorar el acceso a la salud de los colombianos, sino que profundizan las brechas entre segmentos de usuarios con distintas y contrastantes situaciones en cuanto a calidad de la atención, servicios brindados, y oportunidad. Partiendo de los más favorecidos, en un primer nivel, estarían usuarios que suscriben pólizas individuales y 
medicina prepagada; en un segundo, los afiliados del régimen contributivo, dentro de los cuales se diferencian los que tienen plan complementario; en un tercero, los afiliados al régimen subsidiado que cuentan con un plan básico; $y$, en un cuarto, los que carecen de aseguramiento.

En medio de un sector muy segmentado por las oportunidades de negocio -en que una parte apreciable del sector público languidece en la precariedad y la ruina, mientras que otro desarrolla integraciones verticales y otras formas de negocios rentísticos ilegales, reproduciendo arraigadas prácticas especulativas y corruptas-, los logros de algunas clínicas, en certificaciones, integración y coordinación de la cadena, junto a algunos de sus avances investigativos como indicador de innovación, podrían ser importantes en una dinámica futura de configuración del clúster. Se trata de avances más determinados por el acumulado de experiencia y esfuerzo, dentro de firmas específicas, que por acciones conjuntas, con mecanismos de cooperación, interdependencia, generación de confianza y competitividad colectiva, tal como lo plantean los modelos de clúster.

Igualmente, los desarrollos de zonas francas, los pocos procesos de integración productiva que se perciben y la generación de áreas urbanas especializadas, ocurren en torno a grandes y aislados consorcios capitalizados, sin llegarse a conformar un tejido empresarial más denso y localizado. En estos procesos del sector salud, los esfuerzos innovadores se concentran en un reducido número de entidades, y parecen más asociados a la lógica de la firma individual: portafolios de negocios internacionales, certificaciones de calidad y acreditaciones. La persistencia de la fragmentación, y extrema desigualdad de las firmas y entidades, conforman un escenario en el que políticas económicas e iniciativas gremiales avanzan muy poco en las metas de ordenamiento para la competitividad, concertación para alcanzar los plausibles objetivos de optimizar cadenas productivas y desarrollo de clústeres que se han enunciado.

\section{Referencias}

ACHC -Asociación Colombiana de Hospitales y Clínicas-. (2015). Encuesta Impacto de la problemática de flujo de recursos en las clínicas y hospitales afiliadas a la ACHC. ACHC.

ACHC -Asociación Colombiana de Hospitales y Clínicas-. (2018). A 8.2 billones de pesos llegaron las deudas con hospitales y clínicas. http://achc.org.co/a-8-2-billones-de-pesos-llegaron-las-deudascon-hospitales-y-clinicas/

Agudelo, C. A., Cardona, J., Ortega, J. y Robledo, R. (2011). Sistema de salud en Colombia: 20 años de logros y problemas. Ciência \& Saúde Coletiva, 16(6), 2817-2828. http://dx.doi.org/10.1590/ S1413-81232011000600020

Aricapa, R. (2008). Las CTA en el sector Salud. La cadena del dolor. Documentos de la Escuela 72. http:// www.ens.org.co/wp-content/uploads/2016/12/DOCUMENTOS-DE-LA-ESCUELA_72Las-CTA-en-elSector-Salud-La-Cadena-del-Dolor.pdf

Ayala, J. (2014). La salud en Colombia: más cobertura pero menos acceso. Documentos de Trabajo Sobre Economía Regional $n^{\circ}$ 204. Banco de la República. https://doi.org/10.32468/dtseru.204

Bohórquez, F. (2017). Estudio descriptivo de salarios de cargos administrativos y asistenciales 2017. Revista Hospitalaria, 19(116), 4-44.

Cadavid, V., Londoño, S. I., Marín, D. Y. y Peña, S. P. (2016). Análisis de las causas de glosas en la especialidad de ortopedia en la ESE Hospital San Juan de Dios del municipio de Santa Fe de Antioquia en el año 2014 (tesis de posgrado). Universidad CES, Medellín, Colombia. 
Cantillo, D. (2012, 8 de julio). La purga de la San Rafael. El Espectador. https:/www.elespectador.com/ noticias/bogota/purga-de-san-rafael-articulo-358114

Castells, M. (1998). La era de la información. Economía, sociedad y cultura. Alianza.

Congreso de la República de Colombia. (1990). Ley 50 de 1990. Por la cual se introducen reformas al Código Sustantivo del Trabajo y se dictan otras disposiciones. https://www.funcionpublica.gov. co/eva/gestornormativo/norma.php?i=281

Congreso de la República de Colombia. (1993). Ley 100 de 1993. Por la cual se crea el sistema de seguridad social integral y se dictan otras disposiciones. http://www.secretariasenado.gov.co/ senado/basedoc/ley_0100_1993.html

Congreso de la República de Colombia. (2005). Ley 1004 de 2005. Por la cual se modifican un régimen especial para estimular la inversión y se dictan otras disposiciones. http://www.secretariasenado. gov.co/senado/basedoc/ley_1004_2005.html

Congreso de la República de Colombia. (2007). Ley 1122 de 2007. Por la cual se hacen algunas modificaciones en el Sistema General de Seguridad Social en Salud y se dictan otras disposiciones. https://www.minsalud.gov.co/sites/rid/Lists/BibliotecaDigital/RIDE/DE/DIJ/ley-1122-de-2007.pdf

Currie \& Brown. (2018). Estructuración técnica del programa de infraestructura hospitalaria correspondiente al plan de acción "Bogotá Mejor para Todos en Salud", a través del esquema de Asociación Público Privada (APP). http://www.saludcapital.gov.co/Documents/App_4_P_Miguel_ Donovan.pdf

DANE -Departamento Administrativo Nacional de Estadística-. (2016). Boletín técnico Licencias de Construcción - ELIC Diciembre 2015. https://www.dane.gov.co/files/investigaciones/boletines/ licencias/bol_lic_dicl5.pdf

DANE -Departamento Administrativo Nacional de Estadística-. (2018). Estadísticas Vitales ajustadas según métodos demógraficos y estadísticos. Cuadro 1. Tasa de mortalidad infantil por departamento (Defunciones de menores de un año por cada mil nacidos vivos) Serie 20052016. https://www.dane.gov.co/index.php/estadisticas-por-tema/demografia-y-poblacion/ nacimientos-y-defunciones\#tasa-de-mortalidad-infantil

De la Puente, M. (2018). Mercado médico-turístico y movilidad social continental en el segmento de bienestar: caso de Colombia. En F. Giraldo-García et al. (Eds.), Instituciones sociales, conflictos políticos y cambios: Desarrollo y crisis (pp. 179-190). Universidad del Norte.

Deffner, V. y Hoerning, J. (2011). Fragmentation as a Threat to Social Cohesion? A conceptual review and an empirical approach to Brazilian Cities. Committee 21 Sociology of Urban and Regional Development, International Sociological Association, Amsterdam, Países Bajos.

Deloitte. (2014). Healthcare Industry in Turkey. Republic of Turkey Prime Ministry Investment Support and Promotion Agency (ISPAT). https://www.bcct.org.tr/wp-content/uploads/2013-ISPATHEALTHCARE.INDUSTRY.pdf

Dinero. (2016, 7 de julio). Pese a millonaria cartera vencida crece inversión en hospitales y clínicas. https://www.dinero.com/edicion-impresa/negocios/articulo/ aumenta-inversion-en-hospitales-yclinicas-por-fondos-de-capital-privado/225922

Durand, J. (2011). La cadena invisible flujo tenso y servidumbre voluntaria. FCE, UAM. 
El Tiempo. (2016, 28 de febrero). Devaluación acelera inversión extranjera en los hospitales 'top'. https://www.eltiempo.com/archivo/documento/CMS-16523212

El Tiempo. (2017, 28 de diciembre). Los nuevos dueños de Clínica del Country y AutoSnack. https:// www.eltiempo.com/economia/empresas/fusiones-y-adquisiciones-en-colombia-166008

El Tiempo. (2019, 4 de enero). 10 años después, tutelas en salud siguen creciendo en vez de reducirse. https://www.eltiempo.com/justicia/investigacion/ balance-de-tutelas-por-servicios-de-salud-del2018-311250

Eyles, J. (1987). The Geography of the National Health. An Essay in Welfare Geography. Routledge Library Editions.

Ganji, S. (2015). Hub Healthcare: Medical Travel and Health Equity in the UAE. Working Paper 10. http://www.alqasimifoundation.com/admin/Content/File-7122015125423.pdf

Giddens A. (2008). Consecuencias de la modernidad. Alianza.

Gossain, J. (2018, 25 de julio). La verdadera historia de hospitales y clínicas al borde de la quiebra. El Tiempo. https://www.eltiempo.com/vida/salud/ la-verdadera-historia-de-hospitales-y-clinicas-al-borde-dela-quiebra-247988

Harvey, D. (2013). Ciudades rebeldes. Del derecho de la ciudad a la revolución urbana. Akal.

Held, D., McGrew, A., Goldblatt, D. y Perraton, J. (2002). Transformaciones globales. Política, economía y cultura. Oxford University Press.

Hernández, M. (2002). Neoliberalismo en salud: Desarrollo, supuestos y alternativas. En V. M. Moncayo y D. I. Restrepo-Botero (Eds.), La falacia neoliberal: crítica y alternativas (pp. 347-361). Universidad Nacional de Colombia.

Hernández, M. (2004). La fragmentación de la salud en Colombia y Argentina. Una comparación sociopolítica, 1880-1950. Universidad Nacional de Colombia.

Hernández, M. (2017). Consecuencias de la cobertura universal de salud en Colombia. Revista Soberanía Sanitaria, (3), 56-60.

Hervas-Oliver, J. L., Gonzalez, G., Caja, P. y Sempere-Ripoll, F. (2015). Clusters and Industrial Districts: Where is the Literature Going? Identifying Emerging Sub-Fields of Research. European Planning Studies, 23(9), 1827-1872. https://doi.org/10.1080/09654313.2015.1021300

Jaimes, C. y Rincón, L. (2009). Desempeño financiero y económico de las EPS del régimen contributivo $y$ otros actores de la seguridad social en salud en Colombia: 1993-2006. Fundación Universidad de Bogotá Jorge Tadeo Lozano.

Jaramillo-Salazar, H., Latorre-Santos, C., Albán, M. C. y Lopera, C. (2008). El hospital como organización de conocimiento y espacio de investigación y formación. Los recursos humanos en salud y su tránsito a comunidades científicas: el caso de la investigación clínica en Colombia. Universidad del Rosario.

Londoño, J. y Frenk, J. (1997). Pluralismo estructurado: hacia un modelo innovador para la reforma de los sistemas de salud en América Latina. Documento de Trabajo 353. Banco Interamericano de Desarrollo. 
Luhmann, N. (1998). Complejidad y modernidad: de la unidad a la diferencia. Trotta.

Mesa, L. (2018). Configuración del campo de consumo relacionado con la atención en salud en Bogotá, 1980-2014 (tesis de doctorado). Universidad Nacional de Colombia, Bogotá, Colombia.

Ministerio de Comercio, Industria y Turismo. (2009). Competitividad: El desafío para alcanzar un turismo de clase mundial. Documento de política. Ministerio de Comercio, Industria y Turismo.

Ministerio de Comercio, Industria y Turismo. (2016). Decreto número 2147. Por el cual se modifica el régimen de zonas francas y se dictan otras disposiciones. https://incp.org.co/Site/publicaciones/ info/archivos/decreto-2147-mincomercio.pdf

Ministerio de Comercio, Industria y Turismo. (2018). Informe de Zonas Francas - Noviembre de 2017. Comisión Intersectorial Zonas Francas - Secretaría Técnica. https://www.mincit.gov.co/ minindustria/temas-de-interes/zonas-francas

Navarro, M. (2003). Análisis y políticas de clusters: teoría y realidad. Ekonomiaz, (53), 14-49.

OCDE -Organización para la Cooperación y el Desarrollo Económicos-. (2017). Panorama de la santé 2017: Les indicateurs de l'OCDE. Éditions OCDE.

Porter, M. (1991). La ventaja competitiva de las naciones. Plaza y Janés.

Puig, J. y Osorio, V. (2014). El concepto de lo misional permanente y la tercerización ilegal: análisis sociológico y de la jurisprudencia de las Altas Cortes colombianas. Ensayos Laborales $n^{\circ} 22$. Escuela Nacional Sindical.

Quintana, R., Salgado L. y Torres D. (2016). Gestión de Glosas para prestadores de servicios de salud (informe de investigación). Universidad Sergio Arboleda, Bogotá, Colombia.

Sabel, C., Fernandez-Arias, E., Hausmann, R., Rodriguez-Clare, A. y Stein, E. (2012). Export pioneers in Latin America. Inter-American Development Bank, David Rockefeller Center for Latin American Studies and Harvard University. http://dx.doi.org/10.2139/ssrn.2080388

Semana. (2018, 19 de mayo). Las 100 empresas más grandes de Colombia. http://especiales.semana. com/empresas-mas-grandes-de-colombia/2018/listado-cienempresas/index.html

Thouez, J. (1987). Organisation spatiale des systèmes de soins. Presses de l'Université de Montréal.

Touraine, A. (1997). ¿Podremos vivir juntos? Iguales y Diferentes. Fondo de Cultura Económica.

Tullock, G. (2005). The Rent-Seeking Society: The Selected Works of Gordon Tullock. Vol. 5. Liberty Fund.

Vigneron, E. (1999). Santé publique et aménagement du territoire. ADSP, (29) 19. 